\title{
MODELISATION DE LA SURFACE DU FOND DE LA MER, DES OUVRAGES DE PROTECTION DES PORTS ET DES DRAGAGES GENERAUX : LE MODELE BWGEOM
}

\author{
M. VAFIADOU(1): M. SPIRIDAKIS(2) \\ (1) Ingénieur de Recherche, (2) Docteur Ingénieur \\ Marine Technology Development Company SA.- 2nd Merarchias 16. GR -
}

18535 PIRAEUS

\begin{abstract}
BWGEOM is an interactive environment for defining the geometry of breakwaters, navigation channels and general dredging using B-spline curves, $B$ spline patches and Coons' patches. The definition of the bottom surface is based on offsets, i.e. points lying on the sea botfom surface. The definition of breakwaters is based on the bottom surface and a few design parameters, such as the axis of the breakwater, the slopes, the breadth of the crest and the type of the breakwater end. Navigation channels and general dredging are defined using contours and slopes.
\end{abstract}

BWGEOM consists of independed modules, involving the geometry definition of three types of objects, i.e. the cixis, the bottom surface and a third category of objects including breakwaters, navigation channels and general dredging, the drawings of such objects, their intersections with arbitrary planes, as well as calculations based on those objects, for example volume calculations. The modules are connected to each other through a common data-base.

\section{INTRODUCTION}

En génie maritime, la conception d'un nouveau port ou l'aménagement d'un port existant sont des procédures complexes et difficiles. L'examen du point de vue technique et économique de plusieurs solutions alternatives est facilité à notre époque grâce au développement des moyens de calcul.

Le programme BWGEOM présenté ici propose des éléments de solution. Ce système qui fait partie du "C.A.O. for planning and design of harbour works in Greece" developpé chez MARTEDEC. (3). permet la détermination géométrique (par interaction utilisateur/ordinateur) des ouvrages fixes de gravité (briselames), (2), des fonds marins, des chenaux de navigation et des dragages généraux. (voir schéma 1) 


\section{SCHEMA I}

ORGANIGRAMME SCHEMATIQUE

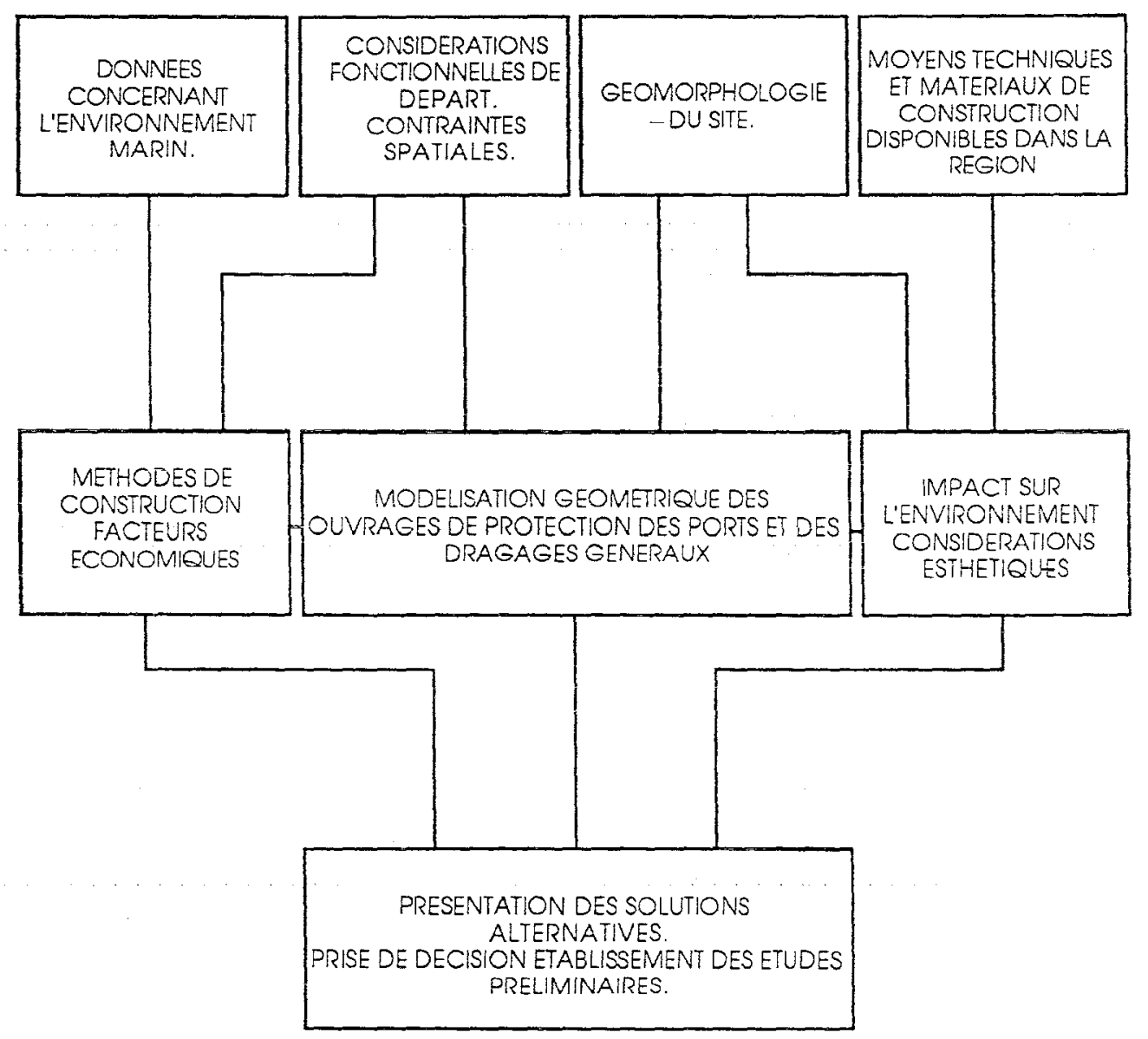

Très peu de travaux ont été effectués jusqu'à maintenant dans ce domaine (4)

\section{LA BASE THEORIQUE DES CALCULS}

\subsection{Définition de la surface du fond de la mer}

La détermination géométrique de la surface du fond de la mer sur un site donné est effectuée par adaptation d'une surface a un ensemble de points (offsets) insérés au programme par un lecteur de courbes.

Les données définissant la surface du fond sont introduites en termes de coordonnées $(x, y, z)$ et la technique des moindres carrés est appliquée pour que l'on puisse adapter une surface mathématique représentant la surface du fond donnée. La surface d' approximation, $z=f(x, y)$, est donnée parl'équation suivante:

$$
z=R(U, v)=\sum_{i=0}^{m-1} \sum_{j=0}^{n-1} M_{i, M}(u) N_{j, N}(v) z_{i, j}
$$


où: $\quad$ MN - ordre de la surface en x.y (spécifié par l' utilisateur) $m, n$ - nombre des points de contrôle (spécifié par l' utilisateur) $u=\left(x-x_{\min }\right) /\left(x_{\max }-x_{\min }\right), v=\left(y-y_{\min }\right) /\left(y_{\max }-y_{\min }\right)$

Mi, M. $\mathrm{Nj}, \mathrm{N}$-fonctions de base, données par les équations suivantes:

$$
\begin{gathered}
M_{i, 1}(x)=\left[\begin{array}{c}
1, \text { si } x_{i} \leq=x<x_{i+1} \\
0, \text { autrement }
\end{array}\right] \\
M_{i, M}(x)=\frac{\left(x-x_{i}\right) M_{i, M-1}(x)}{\left(x_{i+M-1}-x_{i}\right)}+\frac{\left(x_{i+M}-x\right) M_{i+1, M-1}(x)}{\left(x_{i+M}-x_{i+1}\right)}
\end{gathered}
$$

(pour la fonction $\mathrm{Nj}, \mathrm{N}$ les expressions sont équivalentes)

Les fonctions de base sont définies sur des espaces de forme $\left(x_{i}, x_{i}+1\right),\left(y_{j}\right.$, $y j+1)$, afin d'effectuer un contrôle local de la surface d'approximation, car la forme de la surface où un endroit précis dépend seulement des valeurs des points de départ avoisinants à cette région.

\subsection{Définition des brise-lames à talus}

La géométrie des brise-lames est representée par quatre parties en Bspline (intérieure / extérieure / front / partie de clôture) ainsi que par une partie de surface type Coons, correspondant au couronnement de t' ouvrage. La définition est basée sur la géométrie du fond de la mer, sur la forme de l" axe de I' ouvrage, sur les pentes intérieure / extérieure ainsi que sur la forme des extremités de l' ouvrage.

La courbe B-spline a l" expression suivante:

$$
P(t)=\sum_{i=0}^{m-1} P_{i} N_{i, M}(t)
$$

où: $\quad M$ - ordre de la courbe

$m$ - nombre des points de contrôle

$\mathrm{NiM}(t)$ - fonctions de base

et la surface de Coons:

$$
P(u, v)=\begin{aligned}
& (1-u) P_{0}(v)+u P_{1}(v)+(1-v) P_{2}(u)+v P_{3}(u)- \\
& \left((1-u)(1-v) P_{00}+u(1-v) P_{10}+(1-u) v P_{01}+u v P_{11}\right)
\end{aligned}
$$


où: $\quad P_{i}(t), t=u, v, \mathrm{i}=0, \ldots, 3$, sont les quatre courbes de clôture

$P_{i j, ~}^{i}=0,1, j=0,1$ sont les quatre points d'intersection des courbes de clôture

\subsection{Définition des chenaux de navigation et des dragoges généraux}

Les chenaux et les dragages généraux sont définis par la cote de la surface du fond de la mer après dragage, par quatre courbes simples et en caractérisant les pentes de talus. La direction des quatre courbes est présentée sur la Fig. 1. Les paramètres de base pour la détermination des chenaux et des dragages généraux sont présentés dans les fig. 2 (pentes) et Fig. 3 (vue perspective de parois de chenal / dragage). Le programme recherche la partie de la surface du fond où le dragage n'est pas nécessaire c'est-à-dire là où les profondeurs sont plus grandes que la profondeur demandée comme le montre la Fig.4.

\subsection{Définition de la structure des brise-lames ò talus}

La géométrie des différentes couches, correspondant aux divers matériaux de construction des brise-lames, est déterminée en terme de sections-types (Fig.5), dans un système de coordonneés u - v.

Les paramètres nécessaires à la détermination d'une section - type sont les suivants :

- l'épaisseur des couches.

- la cote des couches mesurée à partir du sommet de l'ouvrage.

- les données concernant les types de matériaux de construction ainsi que leur coût.

L'obtention d'une section orthonormée dans le système de coordonnées $x, y z$ du brise-lames est effectuée en utilisant la forme de la section - type, laxe du brise-lames ef le plan de positionnement de la section. Ces paramètres sont utilisés pour le calcul des profondeurs et des épaisseurs des couches de la section réelle.

Il faut mentionner, ici, que le calcul d'une section complète (incluant la géométrie des couches des divers matériaux) peut seulement être effectué pour des sections transversales du brise-lames, sinon seul le contour de la section est calculé. 


\section{LA STRUCTURE DU PROGRAMME}

Le programme BWGEOM se compose de quatre modules de base coptionsniveaux). DEF (Définitions des courbes, de la surface du fond, des brises-lames. de chenaux, et de la géométrie des dragages). DR (Dessins des objets déterminés au niveau DEF). SEC (Intersections entre les objets et des plans quelconques) et CAL (Calcul de différents types comme par exemple calcul des volumes et surfaces, estimation des volumes correspondant aux différentes couches des matériaux des brise-lames). Les modules se trouvent en liaison mutuelle par l'intermédiaire d'une base des données commune. Chaque module est constitué d' un nombre de sous-options exécutant des opérations définies d'avance. La structure du programme ainsi que la communication des modules avec la base des données sont présentées schématiquement dans la Fig 6. La base des données est constituée d'un ensemble de fichiers contenant une information relative au projet. Chaque projet a son propre ensemble de fichiers créés automatiquement dès la première opération de définition du projet. L' information contenue dans la base des données est controlée par un fichier-index, ainsi que par un certain nombre de routines permettant linsertion. le renouvellement et l'obtention de l' information.

\section{CONCLUSIONS ET REMARQUES}

Le système présenté est appliqué actuellement à des cas réels en Grèce. Dès les premiers essais nous avons constaté sa grande utilité dans la procédure d'élaboration des études préliminaires où des configurations alternatives doivent être testées visant l'optimisation techno-économique des projets.

\section{REMERCIEMENTS}

Ce travail a été financé par le programme "Science for Stability" de l' OTAN (Scientific Affairs Division) à qui nous adressons tous nos remerciements.

\section{REFERENCES BIBLIOGRAPHIQUES}

(1) Department of the U.S. Army corps of engineers. "Shore protection manual", 1984.

(2) VAFIADOU M. "Routines for the geometrical modelling of breakwaters and general dredging". internal report. MARTEDEC S A., 1989. 
(3) SPIRIDAKIS M., KOZIS L., KRIKETOS V., PASTRAS C. "Développement d'un système C.A.O. pour les ouvrages de protection des ports". Génie Côtier Génie Civil, Université du Havre. 22-23 Nov. 1990.

(4) WIEBE DE HAAN "Deterministic computer-aided optimum design of rock rubble mound breakwater cross-sections". Coastal engineering, 15 (1991) $3-19$. 


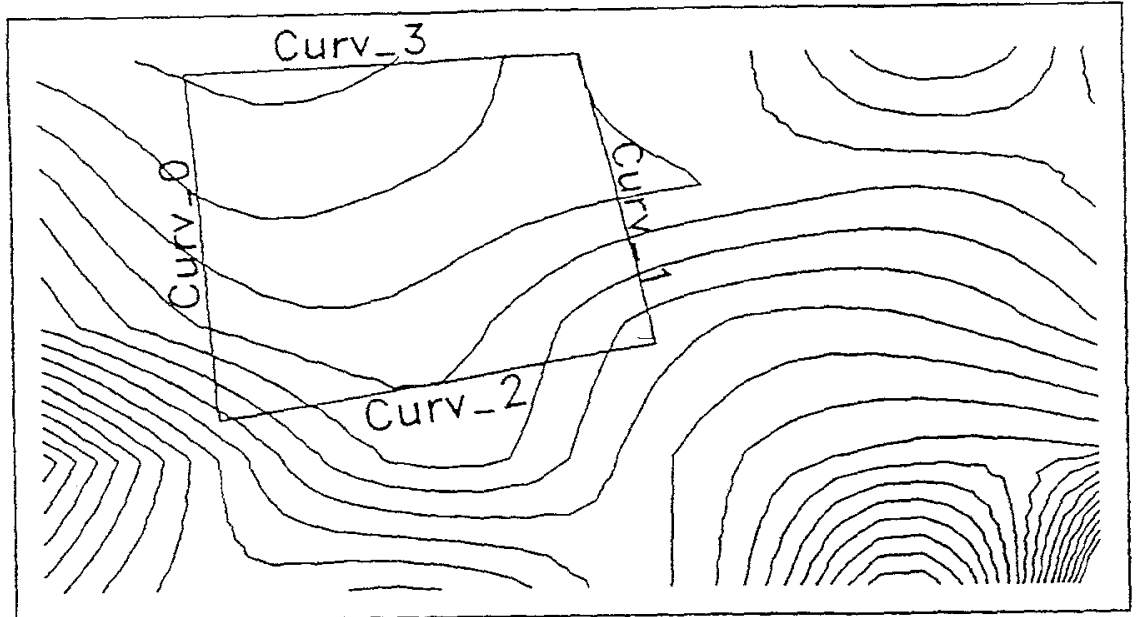

Fig. 1

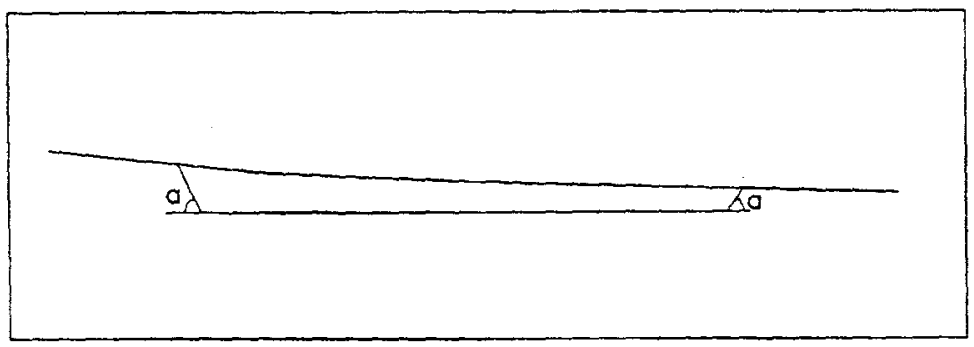

Fig. 2

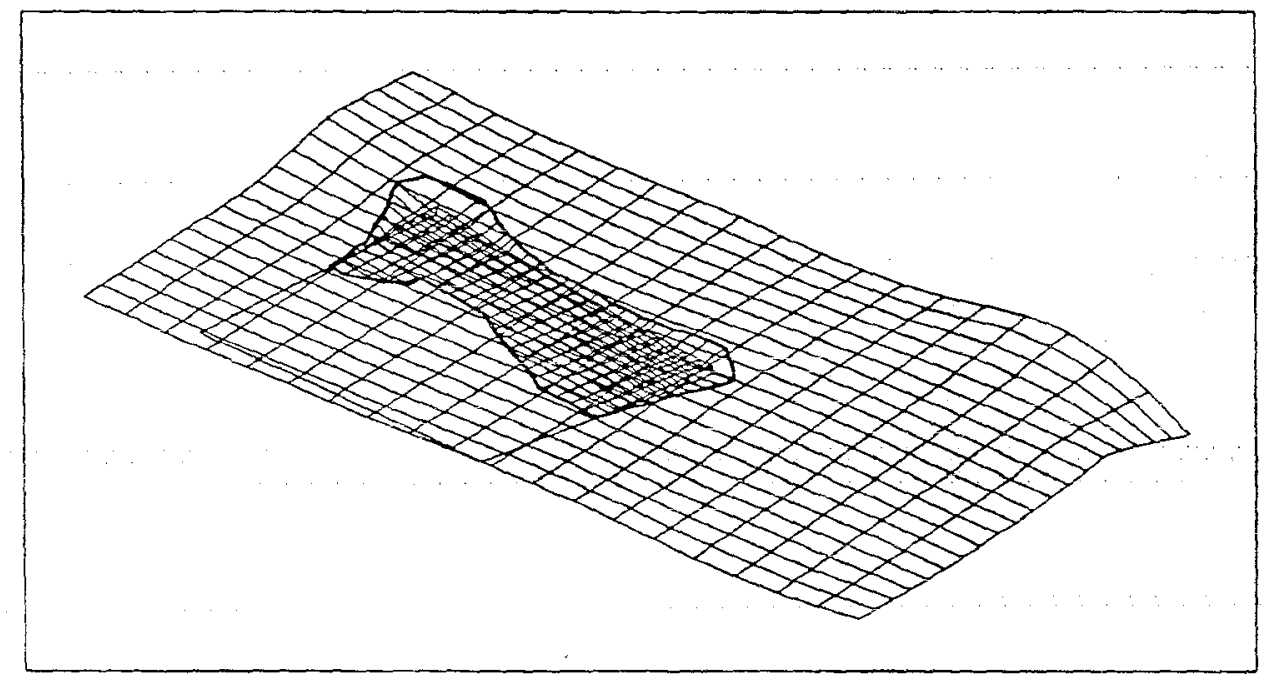

Fig. 3

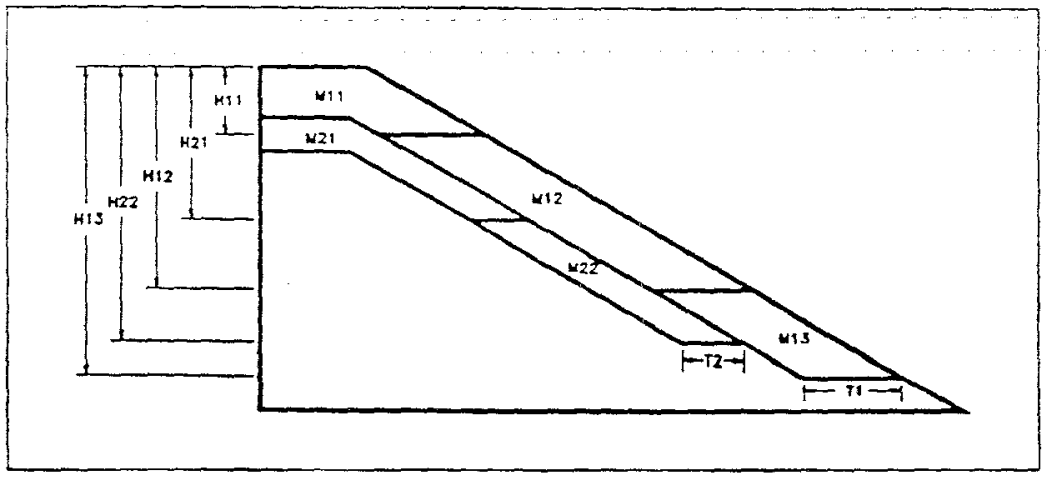

Fig.4 


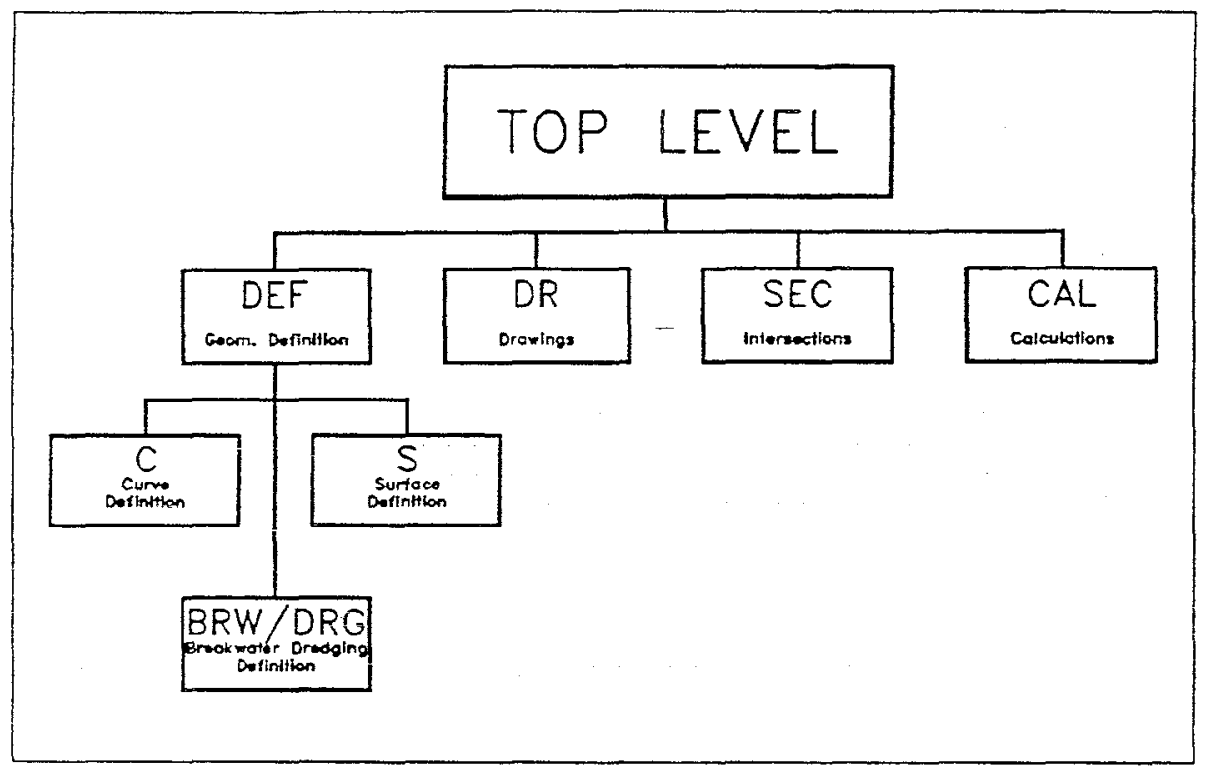

Fig. 5

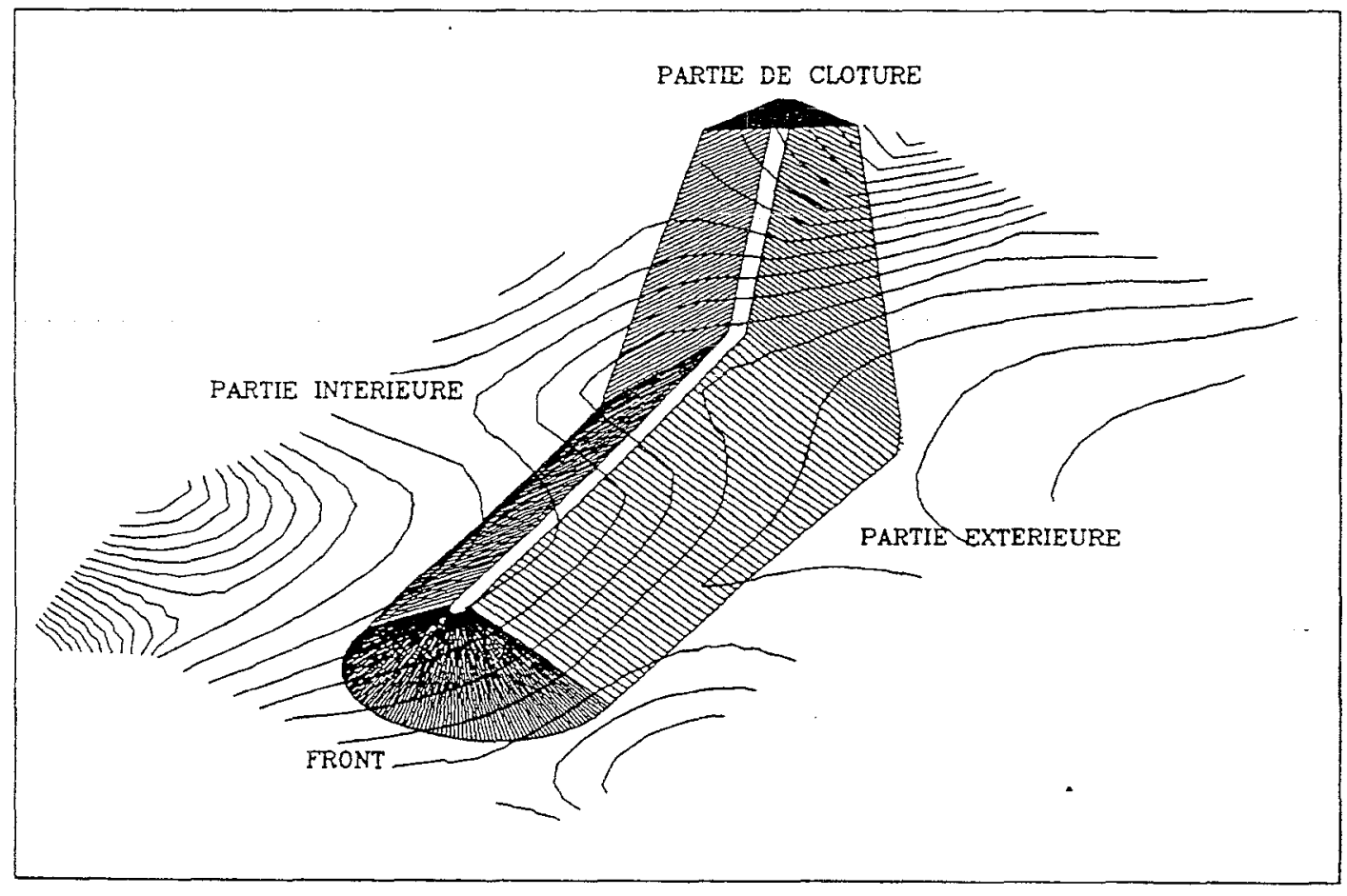

Fig.6

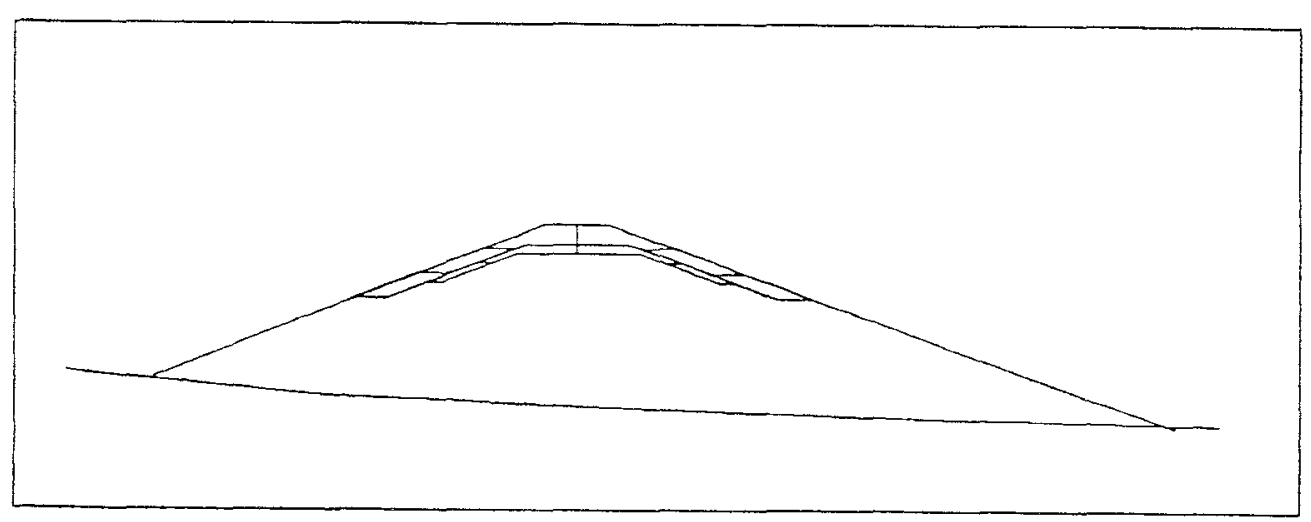

Fin. 7 